\title{
Mimicking Metastases Including Tumor Stroma: A New Technique to Generate a Three-Dimensional Colorectal Cancer Model Based on a Biological Decellularized Intestinal Scaffold
}

\author{
Sarah Nietzer, Dr.rer.nat., ${ }^{1,{ }^{*}}$ Florentin Baur, Dipl.Biol., ${ }^{1, *}$ Stefan Sieber, MSc, ${ }^{1}$ \\ Jan Hansmann, Dr.Ing., Thomas Schwarz, Dipl.Ing., ', Carolin Stoffer, MSc, Heide Häfner, \\ Martin Gasser, Prof.Dr.med., Ana Maria Waaga-Gasser, ${ }^{3}$ Prof.Dr.rer.nat., \\ Heike Walles, Prof.Dr.biol.hum., ${ }^{1,2}$ and Gudrun Dandekar, Dr.Sc.hum. ${ }^{1,2}$
}

Tumor models based on cancer cell lines cultured two-dimensionally (2D) on plastic lack histological complexity and functionality compared to the native microenvironment. Xenogenic mouse tumor models display higher complexity but often do not predict human drug responses accurately due to species-specific differences. We present here a three-dimensional (3D) in vitro colon cancer model based on a biological scaffold derived from decellularized porcine jejunum (small intestine submucosa+mucosa, SISmuc). Two different cell lines were used in monoculture or in coculture with primary fibroblasts. After 14 days of culture, we demonstrated a close contact of human Caco2 colon cancer cells with the preserved basement membrane on an ultrastructural level as well as morphological characteristics of a well-differentiated epithelium. To generate a tissueengineered tumor model, we chose human SW480 colon cancer cells, a reportedly malignant cell line. Malignant characteristics were confirmed in 2D cell culture: SW480 cells showed higher vimentin and lower Ecadherin expression than Caco2 cells. In contrast to Caco2, SW480 cells displayed cancerous characteristics such as delocalized E-cadherin and nuclear location of $\beta$-catenin in a subset of cells. One central drawback of 2D cultures - especially in consideration of drug testing - is their artificially high proliferation. In our 3D tissue-engineered tumor model, both cell lines showed decreased numbers of proliferating cells, thus correlating more precisely with observations of primary colon cancer in all stages (UICC I-IV). Moreover, vimentin decreased in SW480 colon cancer cells, indicating a mesenchymal to epithelial transition process, attributed to metastasis formation. Only SW480 cells cocultured with fibroblasts induced the formation of tumor-like aggregates surrounded by fibroblasts, whereas in Caco 2 cocultures, a separate Caco 2 cell layer was formed separated from the fibroblast compartment beneath. To foster tissue generation, a bioreactor was constructed for dynamic culture approaches. This induced a close tissue-like association of cultured tumor cells with fibroblasts reflecting tumor biopsies. Therapy with 5-fluorouracil (5-FU) was effective only in 3D coculture. In conclusion, our 3D tumor model reflects human tissue-related tumor characteristics, including lower tumor cell proliferation. It is now available for drug testing in metastatic context-especially for substances targeting tumor-stroma interactions.

\footnotetext{
${ }^{1}$ Institute of Tissue Engineering and Regenerative Medicine (TERM), University Hospital of the Julius-Maximilians University, Würzburg, Germany.

${ }^{2}$ Translational Center Würzburg "Regenerative Therapies in Oncology and Musculoskeletal Disease," Fraunhofer Institute Interfacial Engineering and Biotechnology IGB, Würzburg, Germany.

${ }^{3}$ Department of Surgery I, Molecular Oncology and Immunology, University Hospital of the Julius-Maximilians University, Würzburg, Germany.

*Authors contributed equally to this article.

(C) Sarah Nietzer et al., 2016; Published by Mary Ann Liebert, Inc. This Open Access article is distributed under the terms of the Creative Commons Attribution Noncommercial License (http://creativecommons.org/licenses/by-nc/4.0/) which permits any noncommercial use, distribution, and reproduction in any medium, provided the original author(s) and the source are credited.
} 


\section{Introduction}

C ANCER IS A LEADING CAUSE of death worldwide, especially in developed countries, and will gain importance with the progressing age structure of future society. Success in the treatment of this disease depends on the development of appropriate tumor models to improve the translation of novel treatment strategies from research to the patients' situation. Especially in the field of oncology, attrition rates attain up to 95\%. ${ }^{1}$ Reasons are that tumorigenesis is not only regulated intrinsically by genetic changes in the tumor cell itself but also by microenvironmental cues and stromal cell-to-cell interactions that instruct tumor development. ${ }^{2,3}$ These conditions are poorly reproduced by conventional two-dimensional (2D) cell culture models currently used in drug development.

Three-dimensional (3D) cell culture approaches such as Matrigel coating and spheroid cultures have gained importance and substantially improved in vitro tumor models. ${ }^{2,4}$ Tissue engineering offers attractive options for innovative 3D scaffolds that correspond more closely to native tumors than standard 2D cell culture systems or animal models. ${ }^{5}$ However, a crucial step in cancer progression neglected in most models is the invasion across the basement membrane, a process that starts metastasis formation and mostly excludes patients from complete remission under therapy.

Epithelial cancers - known as carcinomas — can become invasive by mainly two different mechanisms: (1) single tumor cell invasion, mostly preceded by an epithelial-to-mesenchymal transition (EMT), through which cells lose epithelial and gain mesenchymal markers and (2) collective tumor cell invasion, probably driven by cancer-associated fibroblasts and independent of EMT. ${ }^{6,7}$ In this study, fibroblasts associate closely with tumor cells, serve as guidance, digest the matrix, and ultimately deposit extracellular matrix (ECM) components to form a complex, tumor-stroma. ${ }^{8,9}$ Metastasis formation shows a reverse program of EMT called mesenchymal-to-epithelial transition (MET). ${ }^{10}$

To generate tissue-engineered tumors, including tumorstroma, in a biological tissue architecture, we use a decellularized part of the porcine jejunum derived from the patented Biological Vascularized Scaffold (DE:302014007893; Bio$\left.\mathrm{VaSc}^{\circledR}\right)$ structure. We define our scaffold as "small intestinal submucosa with preserved mucosa" (SISmuc). It differs from the commonly used SIS scaffolds ${ }^{11}$ by the preservation of the mucosal tissue layer, including crypt, villi, and the basement membrane structures. ${ }^{12}$ This allows us to investigate the process of invasion of tumor cells across the basement membrane into deeper layers of the tissue matrix, a procedure that has been successfully used before in a lung cancer model. ${ }^{12}$

In summary, our aim is to generate an artificial colorectal cancer tissue model. This should be achieved (1) by the integration of a biological tissue scaffold, including basement membrane structures, (2) by the interaction of tumor cells with the tumor stroma, and (3) by applying advanced bioreactor technologies. Our new cancer model paves the way for in vitro analyses of drug efficacy that reflect characteristics of human tumor tissue at different stages of malignancy.

\section{Materials and Methods}

\section{Cell lines and 2D cell culture}

SW480 and Caco2 cells were bought at DSMZ. SW480 cells were cultured in RPMI $+10 \%$ fetal calf serum (FCS) and Caco 2 cells in $\mathrm{MEM}+20 \% \mathrm{FCS}+1 \% \mathrm{NEAA}+1 \%$ Pyruvate. Cells were monitored for pathogen infections at regular intervals. For immunohistochemical 2D stainings, cells were seeded on glass slides until they had reached a confluency of about $70 \%$.

\section{Human fibroblasts}

Fibroblasts were isolated from skin biopsies derived from the University Hospital Wuerzburg (ethics proposal: 182/10).

Skin biopsies were rinsed with phosphate-buffered saline (PBS) with calcium and magnesium $\left(0.5 \mathrm{~g} \mathrm{MgCl}_{2} \cdot 6 \mathrm{H}_{2} \mathrm{O}+\right.$ $0.5 \mathrm{~g} \mathrm{CaCl}_{2} / 5 \mathrm{~L} \mathrm{PBS}$ ) before all fat and tissue remnants were removed. After a second rinsing with $\mathrm{PBS}+$ calcium and magnesium, the skin was cut into strips of $2-3 \mathrm{~mm}$ width. Strips were rinsed with PBS and covered with $2 \mathrm{U} / \mathrm{mL}$ dispase solution (Invitrogen) over night at $4^{\circ} \mathrm{C}$. Then, the dispase solution was removed and $10 \mathrm{~mL}$ PBS with calcium and magnesium $\left(0.5 \mathrm{~g} \mathrm{MgCl}_{2} \cdot 6 \mathrm{H}_{2} \mathrm{O}+0.5 \mathrm{~g} \mathrm{CaCl}_{2} / 5 \mathrm{~L} \mathrm{PBS}\right)$ was added. Epidermis and dermis were separated mechanically. Dermis strips were transferred to a new Petri dish, chopped into small pieces, and incubated in $5 \mathrm{~mL}$ of collagenase solution $(500 \mathrm{U} / \mathrm{mL})$ in a $50-\mathrm{mL}$ tube. The Petri dish was rerinsed with $5 \mathrm{~mL}$ of collagenase solution, which was then put into the 50-mL tube containing the chopped pieces of dermis. They were first incubated for $45 \mathrm{~min}$ at $37^{\circ} \mathrm{C}$ and then centrifuged for $5 \mathrm{~min}$ at $1200 \mathrm{rpm}$. Supernatant was removed and the remaining pellet was resuspended in $2 \mathrm{~mL}$ of culture medium and transferred to a T75 cell culture flask. The next day, $2 \mathrm{~mL}$ of culture medium was added.

\section{SISmuc preparation}

SISmuc was prepared from porcine jejunal segments and consists of small intestine submucosa (SIS) and mucosa (muc). All explantations were in compliance with the German Animal Protection Laws ( $\$ 4$ Abs.3) and the institute's animal protection officer regularly informed the responsible authorities. The animals received proper attention and humane care in compliance with the Guide for Care and Use of Laboratory Animals published by the National Institute of Health (NIH publication no. 85e23, revised 1996) and as approved of by the institutional board of animal protection. After jejunum explantation, the scaffold was prepared as previously described. ${ }^{13-15}$ The SISmuc was generated by removing the mesentery with the vascular tree from the completely decellularized explant.

\section{$3 D$ cell culture of in vitro models}

For static 3D cell culture, cells were seeded onto the SISmuc (see porcine material), fixed between two tailored metal rings - the cell crowns (Fig. 1). All 3D cell cultures were performed under standard conditions $\left(37^{\circ} \mathrm{C}, 5 \% \mathrm{CO}_{2}\right)$ as described before. ${ }^{12,16}$ For monocultures, 100.000 cells in $500 \mu \mathrm{L}$ medium were seeded onto the former luminal side of the SISmuc scaffold. For cocultures, 100.000 tumor cells and 100.000 fibroblasts were seeded together in $500 \mu \mathrm{L}$ medium onto the SISmuc scaffold. After an adhesion time of $2 \mathrm{~h}$, cell crowns with reseeded scaffold were completely filled with medium and cultured for 14 days. Then, the tissue was washed with PBS and fixed in $4 \%$ paraformaldehyde (PFA) for $2 \mathrm{~h}$ followed by paraffin embedding in an 


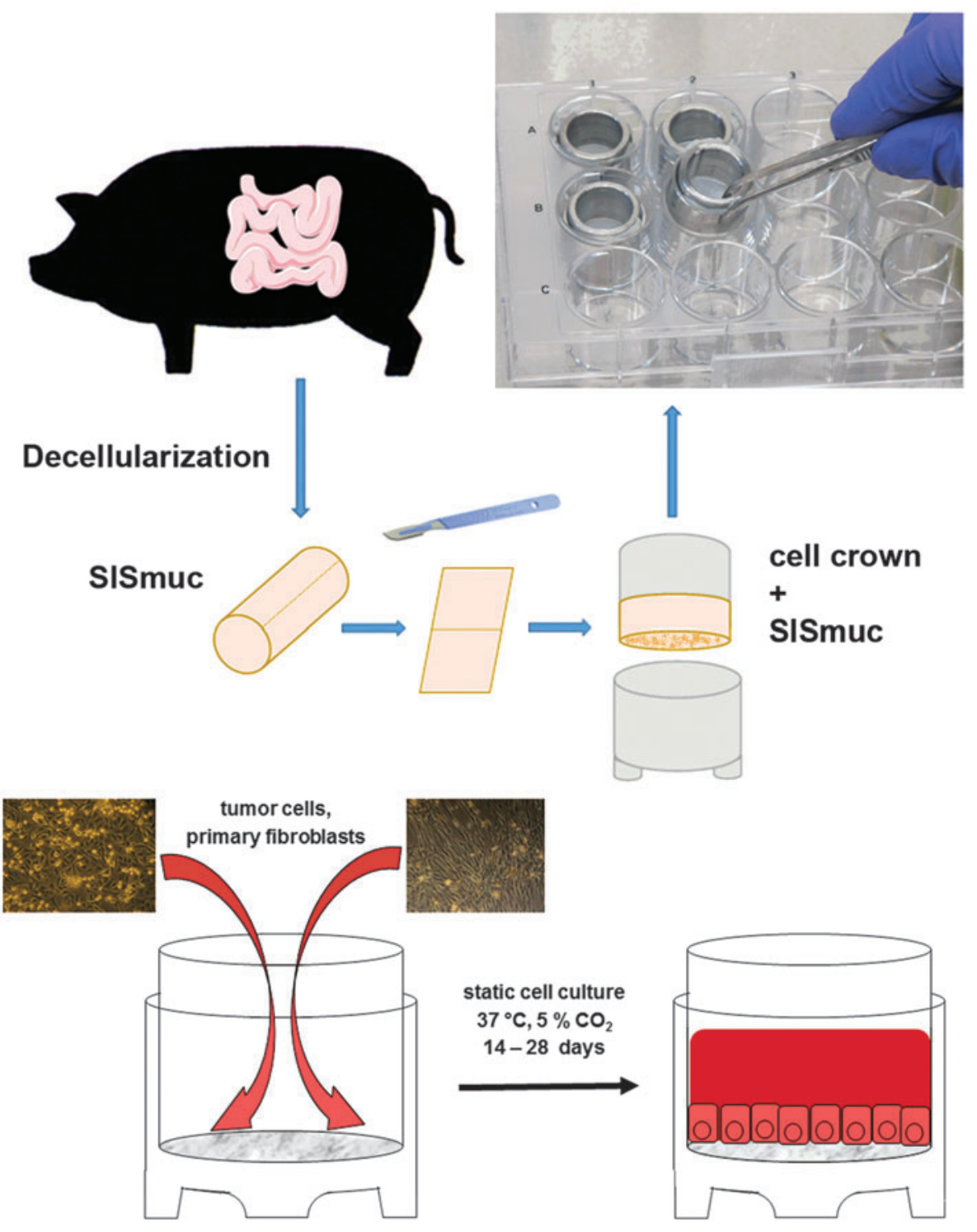

FIG. 1. 3D cell culture in a static system. The SISmuc scaffold derived from a porcine jejunum is fixed between two metal rings, the cell crown. Cells are seeded onto the scaffold and cultured in 12-well plates. For coculturing of tumor cell lines with primary fibroblasts, fibroblasts are isolated from the dermis of human skin biopsies and seeded onto the scaffold together with the tumor cells. 3D, threedimensional; SISmuc, small intestinal submucosa with preserved mucosa. Color images available online at www.liebertpub.com/tec

automated system (STP 120; Thermo Fisher Scientific $\mathrm{GmbH}$ ) for immunohistochemical analysis. Other samples were frozen without any fixation to isolate mRNA from the cells for quantitative real time-PCR (qRT-PCR) analysis.

For dynamic 3D cell culture, SW480 cells were seeded in monoculture and in coculture with dermal fibroblasts onto SISmuc scaffolds and placed in cell crowns 3 days before starting the bioreactor culture (Fig. 2), thus allowing the cells to attach to the scaffold. Afterward, the scaffolds were transferred to the bioreactor and were cultured under dynamic conditions maintaining a constant medium flow for another 10 days. The speed of the blood flow was adjusted to a speed of $3-4 \times 10^{-3} \mathrm{~mL} / \mathrm{min}$ exerting mechanical shear stress on the constructs (Fig. 2D). For the dynamic cell culture, $1 \%$ of Pen/Strep was added to the cell-specific medium.

\section{Bioreactor}

The bioreactor (Fig. 2A) was designed to allow the culture of tissues at the interface between two separated fluid circulation systems. For the design of tailored parts, SolidWorks 2010×64 Edition (Dassault Systemes Deutschland $\mathrm{GmbH}$ ) was used. Construction drawings were sent to GT Labortechnik and parts were manufactured from polysulfone (PSU). To identify an optimal bioreactor setup, different designs were investigated by computational modeling (Fig. 2B, C). For this, COMSOL Multiphysics (Comsol Multiphysics $\mathrm{GmbH}$ ) was used. Bioreactor geometries were transferred to the simulation software, and the fluid domains were parameterized as water at $37^{\circ} \mathrm{C}$. In addition, inlet regions with a specific flow velocity and outlet regions exhibiting ambient pressure conditions were defined. By varying the inlet velocity and by calculating the shear stress distribution, the characterization of a bioreactor design and the identification of the bioreactor operating range were made possible (Fig. 2D).

\section{Stimulation with 5-fluorouracil}

Static models were treated with $25 \mu \mathrm{M}(3.25 \mu \mathrm{g} / \mathrm{mL}) 5$ fluorouracil (5-FU) (Selleckchem) on day 7, 10, and 13. 

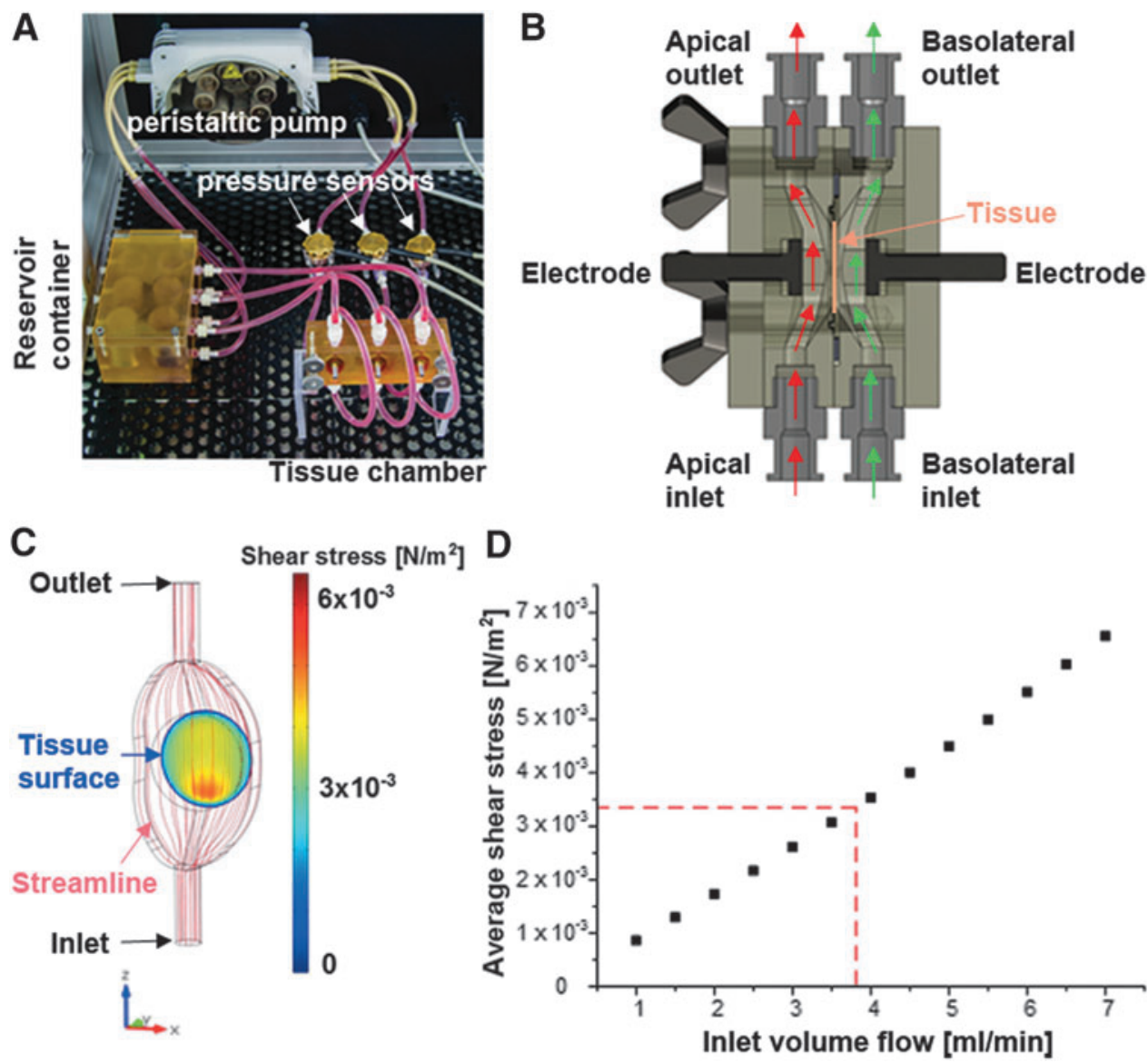

FIG. 2. Compartmented bioreactor for the culture of tissues at the interface between two fluid circulation systems. (A) The bioreactor comprises a tissue chamber, a peristaltic pump, and a reservoir container for the cell culture medium. From the reservoir container, the peristaltic pump delivers cell culture medium to the tissue chamber harboring up to three tissue constructs in separated compartments. (B) A sectional view of such a compartment depicts the path of the flow allowing defined shear stress conditions. On both sides of the tissue, independent fluid circulations ensure apicaland basolateral-specific culture conditions. Stainless steel electrodes support the electrical characterization of the cultured tissue. (C) The streamline and the resulting shear stress on the tissue surface at an inlet volume flow of $3.8 \mathrm{~mL} / \mathrm{min}$ were predicted using computational modeling. Except from a ring of $\sim 50 \mu \mathrm{m}$ at the tissue-bioreactor interface, shear stress values between 3.0 and $5.0 \times 10^{-3} \mathrm{~N} / \mathrm{m}^{2}$ were calculated. (D) By varying the inlet volume flow, the operating range of the bioreactor was characterized to allow the prediction of the average shear stress at a specific velocity of the inlet volume flow. According to the operating range, average shear stress at a volume flow of $3.8 \mathrm{~mL}$ was calculated to $3.34 \times 10^{-3} \mathrm{~N} / \mathrm{m}^{2}$. Color images available online at www.liebertpub.com/tec

Dynamic cultured models were treated on day 7 and 11 of dynamic cell culture with $25 \mu \mathrm{M} 5$-FU. On day 14 of static or dynamic cell culture, tissue was fixed in PFA and embedded in paraffin for immunohistochemistry. Cell Titer Glo Cell Viability Assay (Promega) was performed with 2D cultured cells following the manufacturer's protocol.

\section{Fluorescence immunohistochemistry}

Glass slides were fixed in 4\% PFA for 10 min and SISmuc samples for $2 \mathrm{~h}$. After fixation, glass slides were either directly stained or stored in PBS buffer at $4{ }^{\circ} \mathrm{C}$ for up to 1 week before staining. SISmuc samples were stained as paraffinized sections $(3-5 \mu \mathrm{m})$ and primary antibodies (Ecadherin No. 610181, BD Transduction Laboratories; $\beta$ Catenin No. ab32572, AbCam; Vimentin No. ab92547, AbCam; PCK No. C 2562-.2ML, Sigma-Aldrich Chemie GmbH; Ki67 No. ab16667, AbCam; Collagen IV No. ab6586, AbCam) were diluted 1:100 and incubated over night at $4{ }^{\circ} \mathrm{C}$. Secondary antibodies were diluted 1:400 and incubated for $1 \mathrm{~h}$ at room temperature. Double stainings were performed by using primary antibodies of two different species and secondary antibodies marked with the fluorescent dyes, Alexa-647 or Alexa-555 (Life Technologies $\mathrm{GmbH})$. Nuclei were counterstained by DAPI solved in Mowiol embedding solution. Pictures were taken by using either a confocal laser scanning microscope (SP-8; Leica Microsystems $\mathrm{GmbH}$ ) or a digital microscope (BZ-9000; Keyence Deutschland $\mathrm{GmbH}$ ).

\section{Diaminobenzidine immunohistochemistry}

SISmuc samples were stained as paraffinized sections (3$5 \mu \mathrm{m}$ ) with Villin (\#V1616C01; DCS) according to the instructions of the EnVision+System-HRP (Diaminobenzidine [DAB]) (Dako Deutschland GmbH). 


\section{Quantitative real time-PCR}

We isolated total mRNA from cells using the RNeasy Micro Kit (QIAGEN). The synthesis of cDNA was done by using the iScript Kit (Bio-Rad Laboratories $\mathrm{GmbH}$ ). We used the following primers in a 2-step PCR with the SsoFast $^{\mathrm{TM}}$ EvaGreen ${ }^{\circledR}$ Supermix (BioRad):
Vimentin: CTGGATTTCCTCTTCGTGGA and CGAAAA CACCCTGCAATCTT,

E-cadherin: GACCGGTGCAATCTTCAAAA and CAGGT CTCCTCTTGGCTCTG,

GAPDH: TGACGCTGGGGCTGGCATTG and GCTCTT GCTGGGGCTGGTGG,

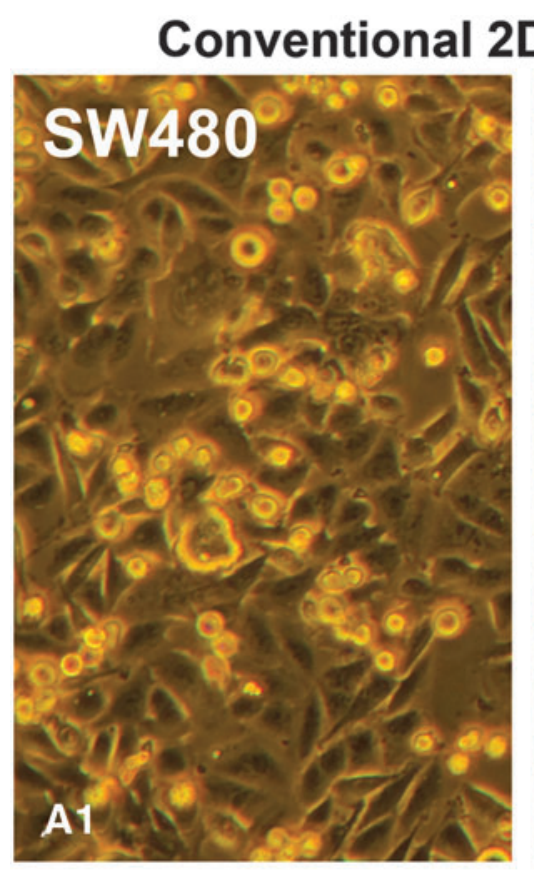

\section{cell culture}

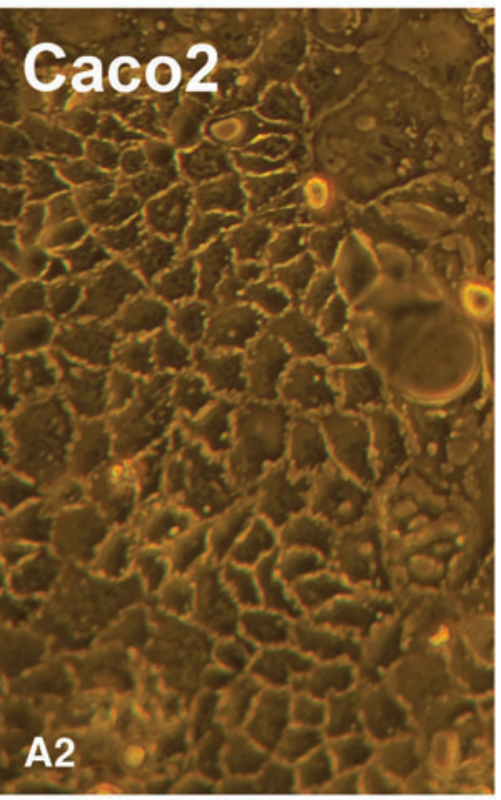

D

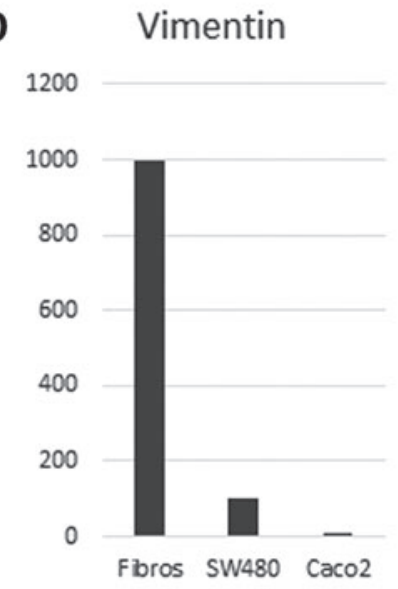

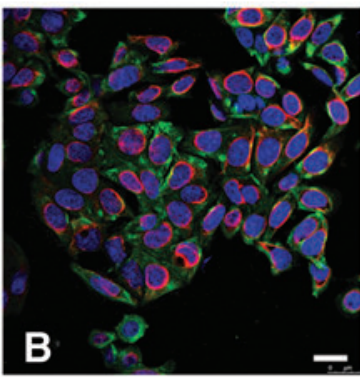
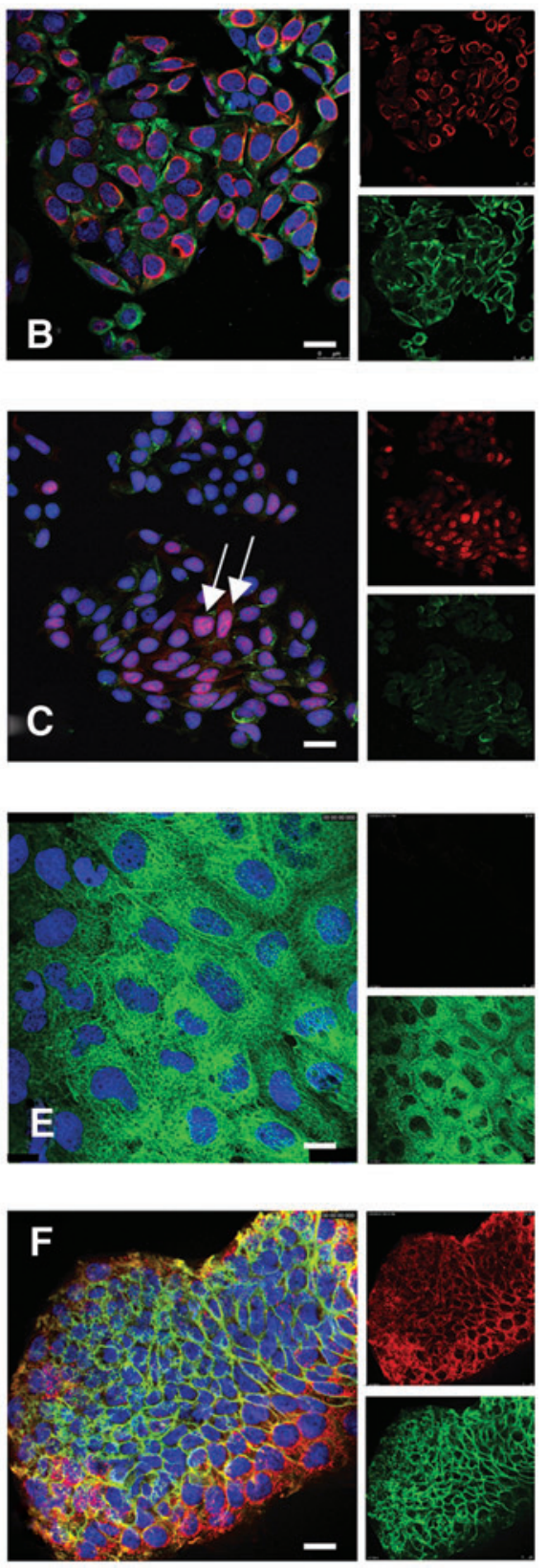

FIG. 3. In conventional 2D cell culture, SW480 cells display a less differentiated and more malignant phenotype than Caco 2 cells. In $2 \mathrm{D}$ cell culture, phase-contrast pictures show that SW480 cells round up and lose contact with the bottom of the culture flask (A1), whereas Caco2 cells display a well-differentiated monolayer (A2). Immunofluorescence double stainings of vimentin (red right top in $\mathbf{B}, \mathbf{E}$ ) and PCK (green right top in $\mathbf{B}, \mathbf{E}$ ) indicate a higher expression of the mesenchymal marker vimentin in SW480 (B) than in Caco 2 cells (E), which was confirmed and quantified by qRT-PCR (D). Immunofluorescence double stainings of the adherence junction protein E-cadherin ( green right bottom in $\mathbf{C}, \mathbf{F}$ ) and $\beta$-catenin (red right top in $\mathbf{C}, \mathbf{F}$ ) demonstrate a lower expression of Ecadherin in SW480 (C) than in Caco2 cells $(\mathbf{F})$. $\beta$-Catenin localizes in the nucleus of several SW480 cells $(\mathbf{C}$, arrows). E-cadherin expression could be also confirmed and quantified by qRT-PCR (D). Scale bars in (B-F): $25 \mu \mathrm{m}$. 2D, two-dimensional; PCK, Pancytokeratin; qRT-PCR, quantitative real time-PCR. Color images available online at www.liebertpub.com/tec 
HPRT: TGACCTTGATTTATTTTGCATACC and CGAG CAAGACGTTCAGTCCT. Genes were normalized to HPRT and GAPDH and the $\Delta \Delta C T$ method was used for evaluation of expression ratios. Primer efficiencies were 91-95\%.

\section{Results}

\section{Caco2 and SW480 colorectal cancer cell lines allow} mimicking of different tumor stages

To establish an in vitro tissue-like 3D tumor model that can simulate tumors in different malignant stages, two different cell lines with reportedly more differentiated (Caco2) or undifferentiated (SW480) growth behavior were tested. Phase-contrast pictures in conventional cell culture showed a stronger adherence to the standard culture flasks in Caco2 cells than in SW480 cells. SW480 cells tended to round up and to separate from the surface, whereas $\mathrm{Caco} 2$ cells built a tight monolayer (Fig. 3A1, A2). Compared to Caco2 cells, the more malignant SW480 cells showed a higher expression of vimentin in immunofluorescence staining, thus indicating an initiated EMT process going along with a lower expression of the adherence junction marker E-cadherin. This was confirmed quantitatively by qRT-PCR (Fig. 3D). We noted that in some of the SW480 cells, but not in any Caco 2 cells, $\beta$-catenin was localized within the nucleus
(Fig. 3C, arrows), indicating a more malignant state of SW480 cells. ${ }^{16}$

\section{Generation of $3 D$ tumor models}

For $3 \mathrm{D}$ in vitro colon cancer tumor model development, we applied the SISmuc as a scaffold with intestinal microarchitecture as used before for artificial tissue and lung tumor models. ${ }^{12,14}$ First, we determined a cell number of $1 \times 10^{5}$ Caco 2 cells and a culture period of 14 days on our SISmuc scaffold as being optimal to generate artificial tissue with epithelial characteristics. We could demonstrate differentiation progress by immunohistochemical staining of villin. This protein involved in absorption was basolaterally located after 7 days of culture (Fig. 4A) and moved to the apical surface of the epithelial monolayer after 14 days of culture (Fig. 4B). Furthermore, transmission electron microscopy (TEM) analysis revealed the formation of regular microvilli, close cell-to-cell contacts with apical tight junctions (arrow in Fig. 4d), the presence of storage vacuoles, ${ }^{17}$ and a close attachment to the preserved basement membrane of the decellularized intestinal scaffold (Fig. 4CE). The same culture conditions that allowed welldifferentiated growth of Caco 2 cells were applied to SW480 cells in the following experiments as well as to the coculture approaches with primary fibroblasts.
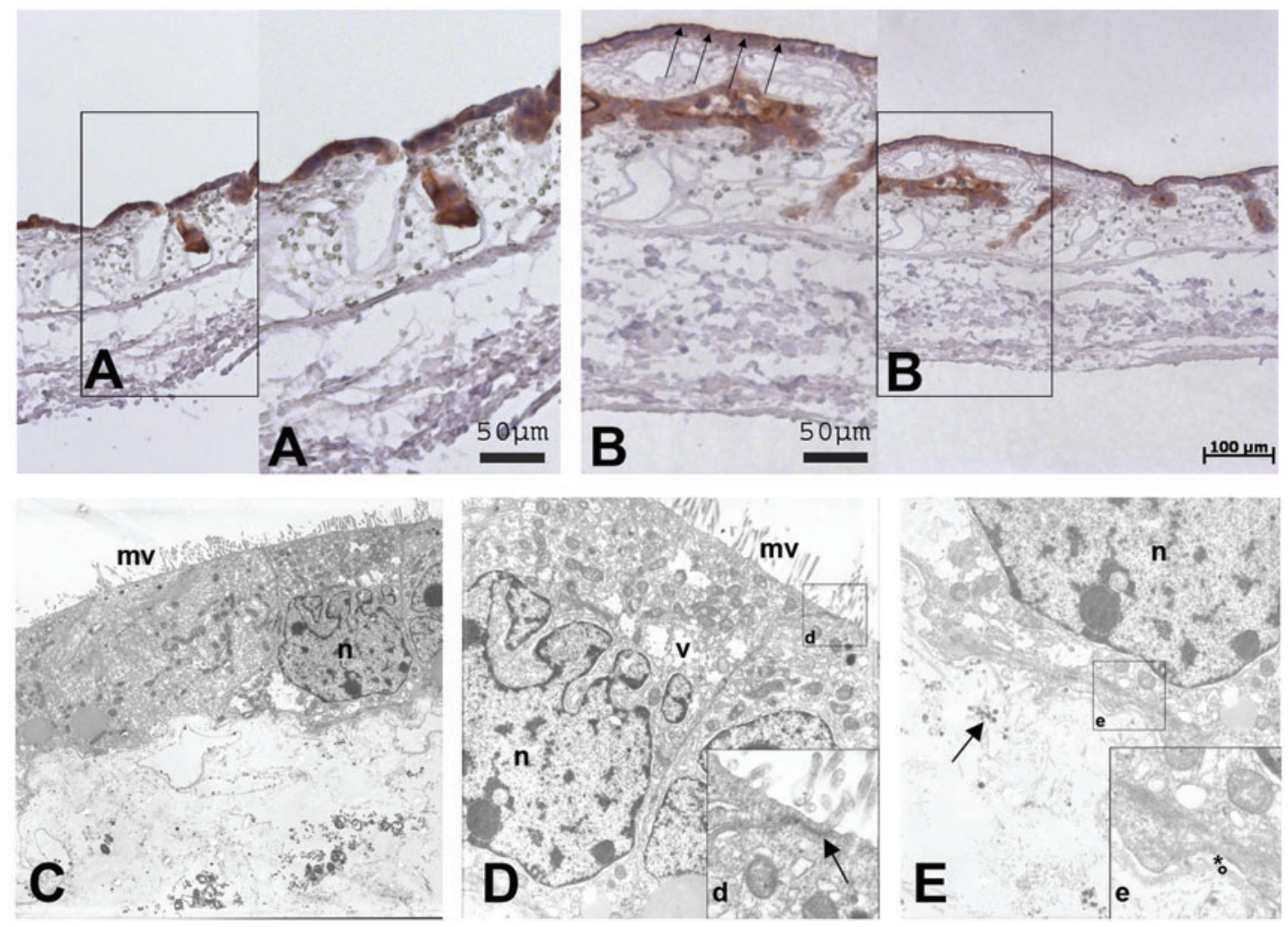

FIG. 4. On the 3D SISmuc scaffold, differentiation of Caco2 cells is achieved after 14 days of culture. Immunohistochemical DAB-staining of villin on paraffin sections shows a basolateral/cytoplasmic location after 7 days of cell growth (A) and its change to the apical side after 14 days of growth (B, arrows). Ultrastructural analysis of Caco 2 cultured on SISmuc for 14 days displays a closed monolayer on top of the SISmuc. The cells show a well-differentiated morphology of microvilli (C, D: mv), the formation of close apical tight junctions (arrow in d) as well as mucin-vacuoles ( $\mathrm{v}$ in D). The nucleus is marked with n. Moreover, cells attach to the preserved basement membrane $\left(*=\right.$ lamina lucida ${ }^{\circ}=$ lamina densa) of the SISmuc (e). Arrow in (E): collagen fibers. DAB, diaminobenzidine. Color images available online at www.liebertpub.com/tec 
The percentage of proliferating cells in $3 D$ cell culture resembles the in vivo situation

In our 3D in vitro tumor model for colorectal carcinoma, we were able to show a clearly decreased proliferation by immunohistochemical Ki67 staining. Compared to almost 100\% Ki67-positive cells in conventional 2D cell cultures of Caco2 and SW480 cells (Fig. 5A, B), lower cell proliferation appeared in 3D cultures of both tumor cell lines (Fig. 5C, D). The percentage of proliferating cells in patients' biopsies from different tumor stages in the tumor center and border ranged from $6 \%$ in UICC I, $16 \%$ in UICC II, $32 \%$ in UICC III, to $35 \%$ in UICC IV. To find out if markers from 2D stainings were maintained in 3D and could be aligned to morphological growth patterns indicating invasion or metastasis formation, we investigated tissue morphology by hematoxylin and eosin (HE) staining and stained for molecular EMT markers such as Pan-cytokeratin (PCK) and vimentin, epithelial differentiation (E-cadherin), and nuclear $\beta$-catenin localization.

Tissue microenvironment and fibroblast coculture enhance differences of tumor characteristics in $\mathrm{Caco} 2$ and SW480 cells

As demonstrated by $\mathrm{HE}$ stainings, Caco2 cells formed a monolayer on top of the scaffold (Fig. 6A). Primary fibro- blasts exhibited a flat cell morphology and grew inside the scaffold. The former villi topography of the SISmuc was remodeled to a plain surface (Fig. 6B). In contrast to the Caco 2 cells, more malignant SW480 cells grew chaotically without the formation of a closed monolayer and migrated more deeply into the former crypt and vessel structures of the gut. Moreover, single SW480 cells completely lost contact with the scaffold (Fig. 6C). The immunofluorescence analysis of vimentin and PCK showed a high expression of $\mathrm{PCK}$ in $\mathrm{Caco} 2$ cells and vice versa, a high expression of vimentin in fibroblasts (Fig. 6D, E). We noted that in contrast to $2 \mathrm{D}$ conditions, vimentin could no longer be detected in SW480 cells cultured on SISmuc (Fig. 6F, compare to inset). In our coculture model, we seeded the same number of tumor cells and fibroblasts at the same time onto the SISmuc scaffold. Interestingly, fibroblasts from the same batch affected Caco2 and SW480 cell growth and morphology differently. After 2 weeks in coculture with fibroblasts, Caco 2 cells formed multilayers in single areas. However, HE staining revealed no morphological changes compared to monocultures (Fig. 6G). On the contrary, fibroblast coculture showed a strong morphological impact on SW480 cells and resulted in the formation of tumor-like nodes (Fig. 6H). Cocultures with both cell lines showed less protruding villi structures similar to fibroblast monocultures.
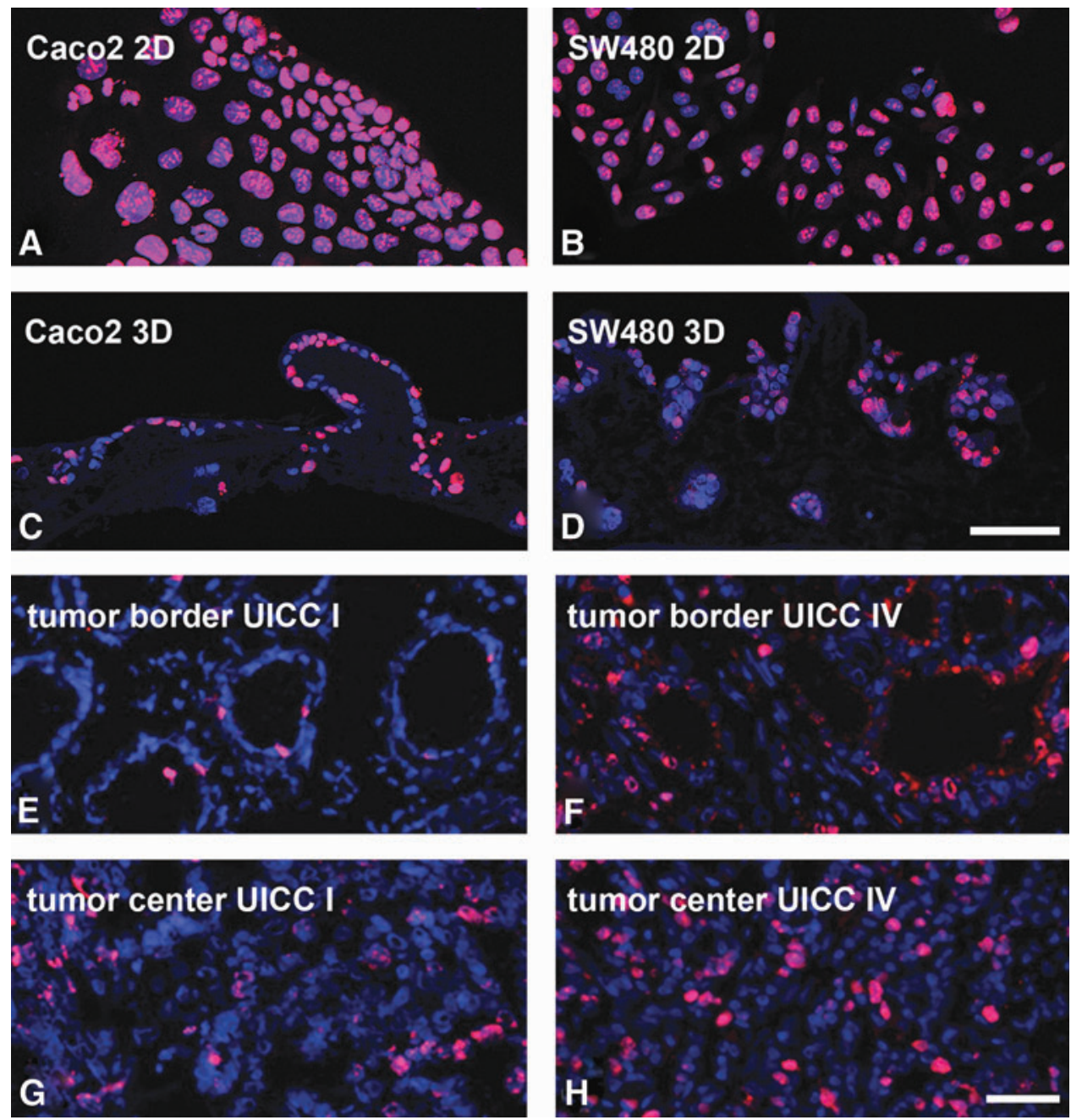

FIG. 5. The amount of proliferating cells in the 3D in vitro tumor model decreases compared to conventional 2D cell culture and correlates better to low-grade (UICC I) and high-grade (UICC IV) tumors. Ki67 immunofluorescence staining of Caco 2 cells (A) and SW480 cells (B) in 2D cell culture demonstrates a high proliferation that decreases distinctly in 3D cultures of $\mathrm{Caco} 2$ (C) and SW480 cells (D). The number of proliferating cells cultured under 3D culture conditions correlates more precisely with those found in tumors with a low $(\mathbf{E}, \mathbf{G})$ and high grade $(\mathbf{F}, \mathbf{H})$ of malignancy. Pictures were taken from the tumor border $(\mathbf{E}, \mathbf{F})$ as well as from the center of the tumor $(\mathbf{G}, \mathbf{H})$. Scale bar in (D) represents $75 \mu \mathrm{m}$ for (A-D). Scale bar in (F) represents $50 \mu \mathrm{m}$ for $(\mathbf{E}-\mathbf{H})$. Color images available online at www.liebertpub.com/tec 


\begin{abstract}
FIG. 6. Fibroblasts induce tumor cell aggregation of SW480 on the SISmuc scaffold. HE staining of paraffin sections of the $3 \mathrm{D}$ in vitro tumor model shows that $\mathrm{Caco} 2$ cells grow as a monolayer (A), while SW480 cells exhibit a less differentiated phenotype with loose cell-to-cell contacts (C). Within both cell lines, former crypt (c) and villi (v) structures remain clearly visible. In contrast to this, fibroblasts remodel the matrix to a flat layer without villi structures (B). PCK/vimentin double immunofluorescence staining demonstrates a strong PCK staining of $\mathrm{Caco} 2$ and SW480 cells (D, F), and a high expression of vimentin in fibroblasts (E). Vimentin expression is lost in SW480 in 3D culture conditions compared to 2D conditions (F, inset: 2D staining: PCK/vimentin). HE staining of cocultures indicates a preserved monolayer formation of $\mathrm{Caco} 2$ cells even though in some parts, multilayers could also be observed $(\mathbf{G})$. In SW480 cocultures, the morphology of tumor cells changed to tumor tissue-like aggregates inside the matrix (H, arrows). Immunofluorescence double staining of $\mathrm{PCK} /$ vimentin shows that in $\mathrm{Caco} 2$ cocultures, the fibroblasts stay in the bottom compartment with the tumor cells on top (I), whereas in SW480 cocultures, the tumor cells are enclosed by fibroblasts that grow on top, beneath, and between the tumor nodes $(\mathbf{J})$. HE, hematoxylin and eosin. Color images available online at www.liebertpub $. \mathrm{com} / \mathrm{tec}$
\end{abstract}
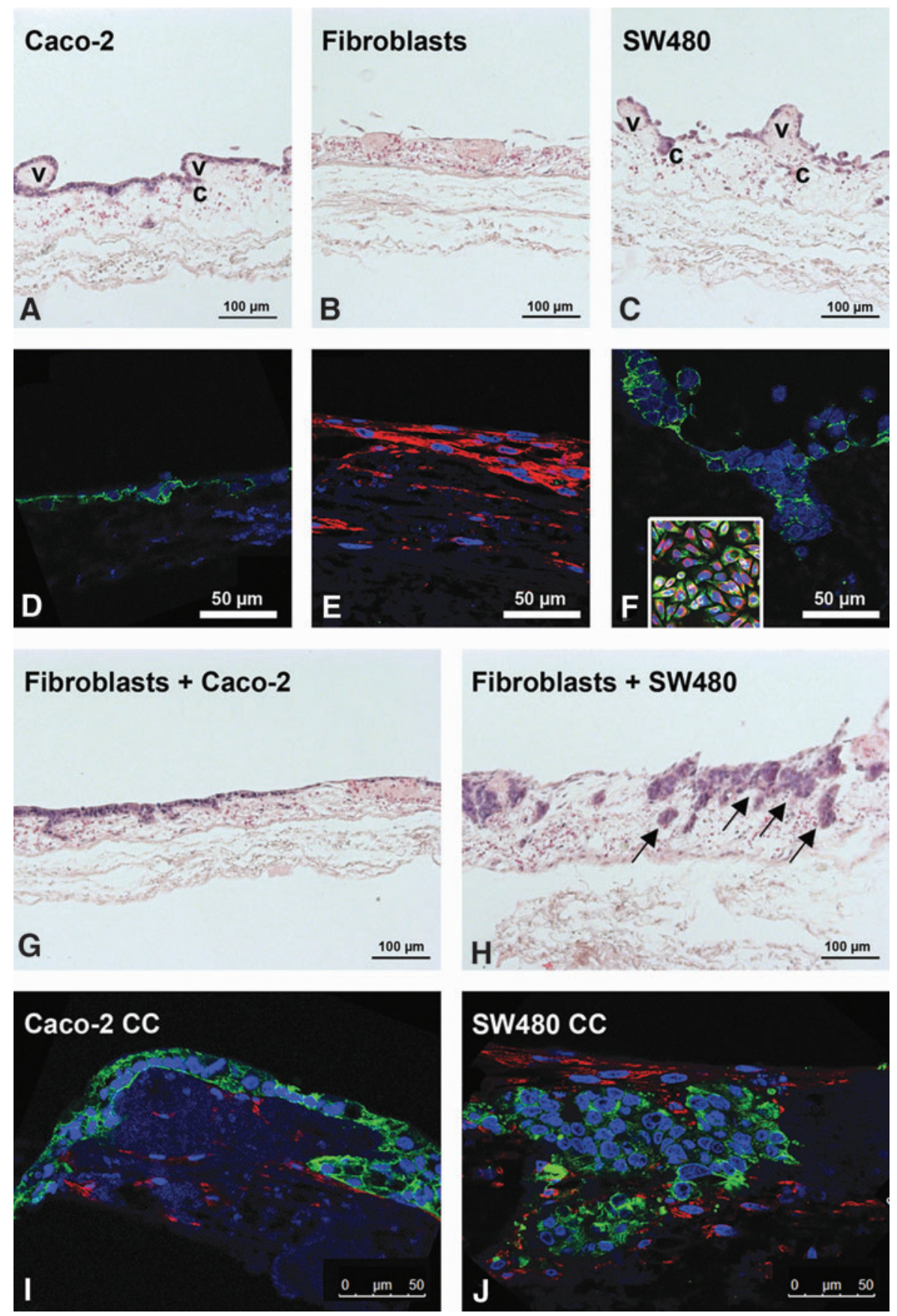

Evidently, neither the seeding order nor a higher ratio of fibroblasts impaired the overall coculture morphologies (data not shown). Depending on the tumor cell type, PCK/ vimentin fluorescence double staining demonstrated that fibroblasts and tumor cells were located in different regions of the SISmuc in 3D cell culture (Fig. 6I, J). In Caco2 cocultures, fibroblasts accumulated inside the matrix, clearly separated from the tumor cells on top (Fig. 6I). In SW480 cocultures, fibroblasts grew on the surface of the matrix, at the interface separating mucosa and submucosa, between the tumor-like nodes and also in close association to them (Fig. 6J). SW480 and fibroblasts could be distinguished by morphological features and by vimentin staining, since downregulation of vimentin was maintained in SW480 coculture settings.

\section{Epithelial characteristics of SW480 cells are more prominent in the $3 D$ microenvironment}

Next, we correlated our morphological observations to the expression of E-cadherin as a MET-marker and to the expression of $\beta$-catenin. As expected, we could show by confocal analysis of E-cadherin/ $\beta$-catenin immunofluorescence double staining that both colocalize in $2 \mathrm{D} \mathrm{Caco} 2$ monocultures at the cell junctions. This was not changed by the coculture with fibroblasts in 3D (Supplementary Fig. S1; Supplementary Data 

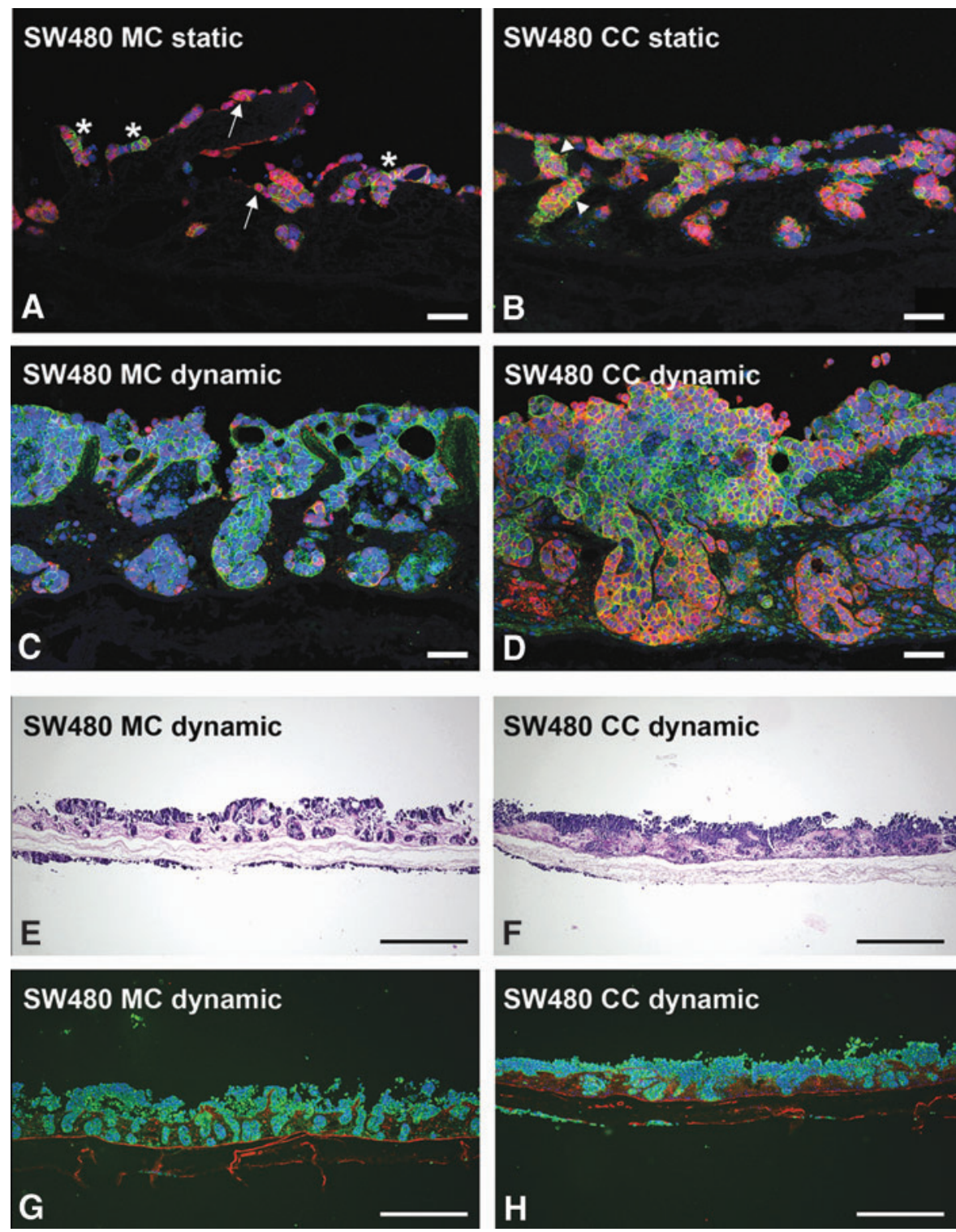

FIG. 7. In coculture with fibroblasts, the crypt structure of the SISmuc is changed by the SW480 tumor cells. In static SW480 monoculture, $\beta$-catenin (red in AD) localizes in the cytoplasm and the nucleus of several cells (arrows in A). E-cadherin (green in A-D) staining shows single areas of strong expression within cell aggregates (stars in $\mathbf{A})$. In static coculture of SW480 and fibroblasts, E-cadherin staining is more pronounced, especially in SW480 aggregates, and is partly colocalized with $\beta$-catenin (arrowheads in $\mathbf{B}$ ). Under dynamic culture conditions in the flow bioreactor, this effect is enhanced in the coculture (D) compared to monoculture (C). While in monoculture, SW480 tumor cells fill the former crypts of the SISmuc scaffold and preserve the regular structure of the gut, the former crypt structure is remodeled by the cells in coculture with fibroblasts (D). This effect is more visible in $\mathrm{HE}$ staining $(\mathbf{E}, \mathbf{F})$ as well as in a double staining of PCK (green in $\mathbf{G}, \mathbf{H}$, tumor cells) and collagen-IV (red in $\mathbf{G}, \mathbf{H}$, basement membranes). Scale bars in (A-C) represent $75 \mu \mathrm{m}$. Scale bars in (E-H) represent $500 \mu \mathrm{m}$. Color images available online at www.liebertpub.com/tec are available online at www.liebertpub.com/tec). In contrast to that, a weaker expression of E-cadherin and concentration in areas of cell aggregates were found in SW480 monocultures (Fig. 7A, stars). In coculture, E-cadherin accumulated in tumorlike nodes at the cell borders (Fig. 7B). $\beta$-Catenin expression in mono- and cocultures was more pronounced in SW480 cells than in Caco2 cells (compare Supplementary Fig. S1). Moreover, in SW480 monocultures, $\beta$-catenin was observed in some single cells inside the nucleus (Fig. 7A, arrows), which was also visible in $3 \mathrm{D}$ coculture samples (Fig. 7B). In addition, in several other areas of the artificial tumor tissue generated by SW480 cells and fibroblasts, a yellow staining indicative of colocalization appeared in the merged staining of E-cadherin and $\beta$-catenin (Fig. 7B arrowheads).

\section{Dynamic cell culture conditions support tumor tissue generation and association with the tumor-stroma}

To ensure an optimal in vivo-like nutrient supply and waste removal, we applied dynamic culture conditions to
SW480 mono- and cocultures using a newly constructed flow bioreactor system (Fig. 2). These conditions enhanced SW480 tumor cell growth during a culture period of 14 days. As a result, SW480 filled all the former crypts and built multilayers on the former villi (Fig. 7C, E, G). In contrast to that, SW480 cocultured with fibroblasts remodeled the SISmuc and changed the crypt structure as shown by E-cadherin/ $\beta$-catenin double staining (Fig. 7D) as well as HE staining (Fig. 7F) and PCK/collagen-IV double staining (Fig. $7 \mathrm{H})$. E-cadherin staining displayed quite a homogenous distribution within the areas of cells tightly packed together in monoculture (Fig. 7C) as well as in coculture (Fig. 7D). In contrast to the dynamic SW480 monoculture, where single cells express $\beta$-catenin in the cytoplasm, the majority of cells in the dynamic coculture setting expressed $\beta$-catenin only at the cell borders (Fig. 7D). The formation of a tumor-like tissue with a surrounding tumor stroma was demonstrated by PCK/vimentin double staining to differentiate between tumor tissue and surrounding fibroblasts (Fig. 8). Tumor cell aggregates were 


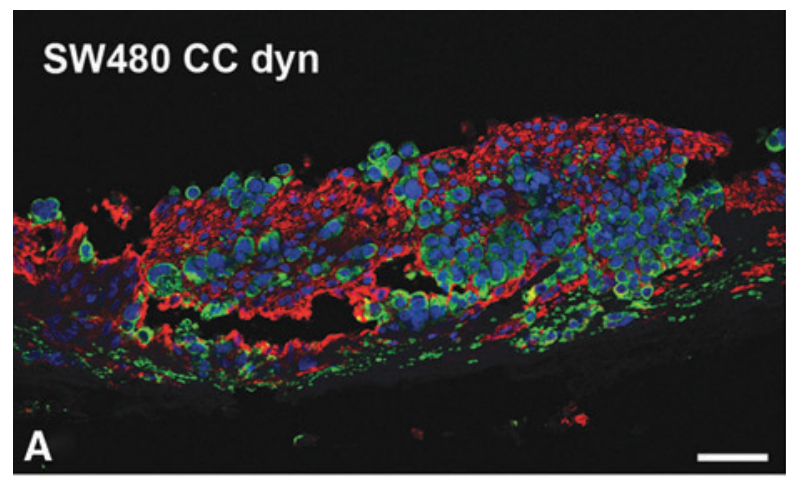

FIG. 8. The 3D colon cancer model with integrated fibroblasts phenocopies a patient's colon adenocarcinoma. PCK (green) and vimentin (red) fluorescence double staining demonstrates a close association of SW480 tumor cells (PCK positive) with cocultured fibroblasts (vimentin positive) in a dynamic bioreactor culture. This phenotype reflects the morphology of a colon adenocarcinoma from a patient's biopsy (C). Scale bars: $50 \mu \mathrm{m}$ in $\mathbf{( A , C )}$ and $100 \mu \mathrm{m}$ in $\mathbf{( B )}$. Color images available online at www.liebertpub.com/tec
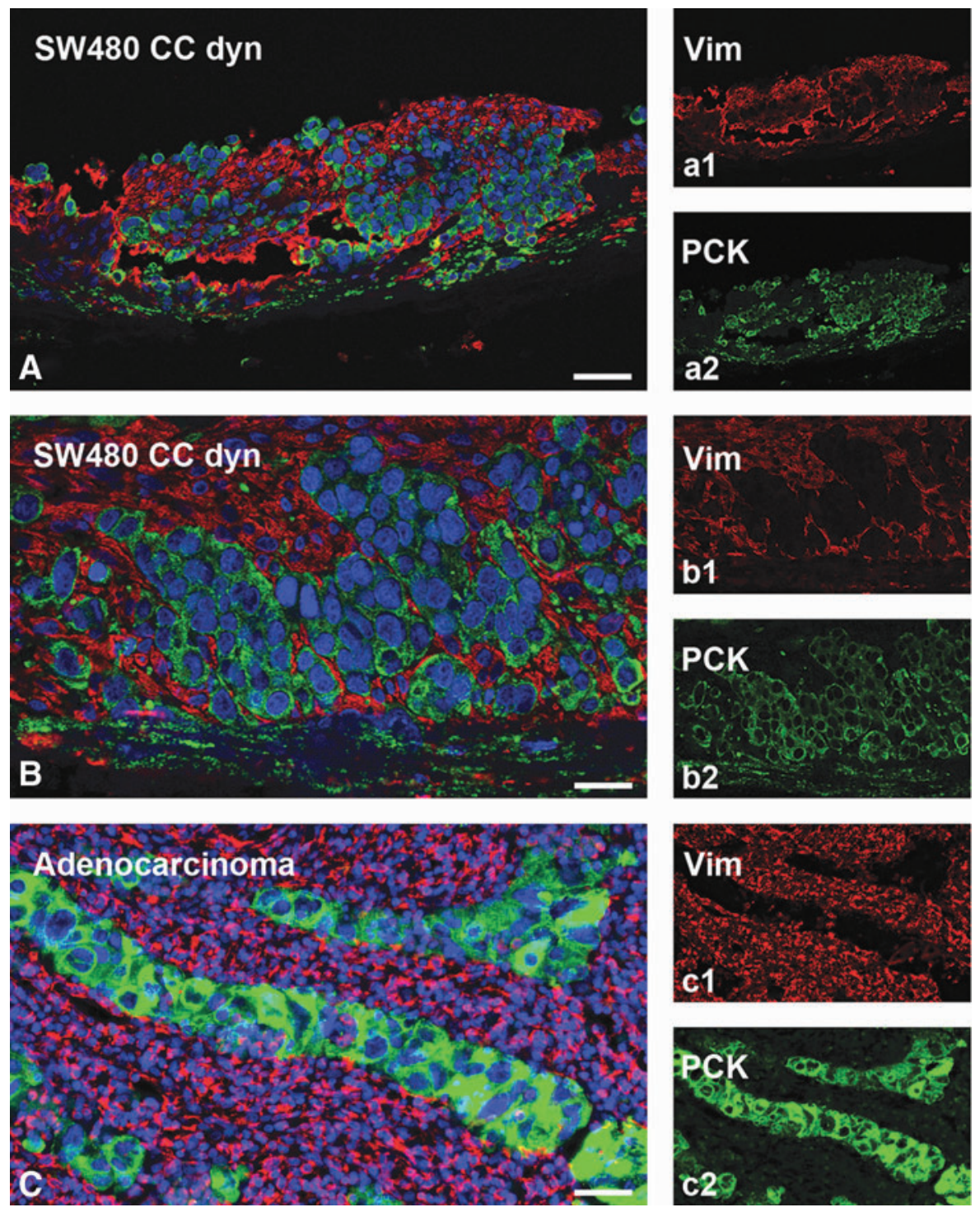

enclosed by fibroblasts and tumor cells completely overgrew the SISmuc in several parts of the scaffold (Fig. 8A, B). The morphology of the generated tissue closely resembled that of a colon adenocarcinoma (Fig. 8C).

\section{Fibroblasts remodel basement membrane structures under dynamic cell culture conditions}

To analyze basement membrane structures, we stained dynamically cultured SW480 mono- and cocultures for collagen-IV. The tumor cells alone (marked by PCK, green in Fig. 9) did not change the collagen structure of the SISmuc (Fig. 9A-C). Around former crypts, basement membranes are precisely visualized by anticollagen-IV staining and mostly well preserved (Fig. 9B, arrows). In coculture with fibroblasts (Fig. 9D-F), these distinct borders are interrupted (Fig. 9E, arrow) and the red collagen-IV staining shows a thickened structure of the SISmuc (Fig. 9E, F). Fibroblasts are able to remodel the SISmuc alone (Fig. 9G, $\mathrm{H})$ as well as in coculture with SW480 (Fig. 9D-F). In coculture, fibroblasts (stained by vimentin, red in Fig. 9J, K) intermingle with the tumor cells (stained by PCK, green in
Fig. 9J, K) and, in some areas, seem to lead them deeply into the collagen structure of the SISmuc (Fig. 9K).

\section{5-FU treatment reduces tumor cells under static and dynamic conditions in coculture settings with primary fibroblasts}

SW480 cells were nearly insensitive to 5-FU under 2D culture conditions (Supplementary Fig. S2A) and static 3D monocultures (Supplementary Fig. S2C). In contrast to this, the treatment of cocultures with $25 \mu \mathrm{M}$ 5-FU resulted in decreased tumor cell numbers as clearly shown by a PCK/vimentin staining under 3D static culture conditions (green in Fig. 10A, C), as well as under 3D dynamic culture conditions (Fig. 10C, D). In fibroblasts, we observed no effect of this treatment in monocultures (red in Supplementary Fig. S2C) and in cocultures (red in Fig. 10B, D). In coculture of SW480 with fibroblasts, the dense clusters of tumor cells surrounded by fibroblasts shrank and became less compact after a treatment period of 1 week. In the untreated controls, many living cells were flushed into the medium reservoir, but we found only dead tumor cells in the medium reservoir of bioreactors 

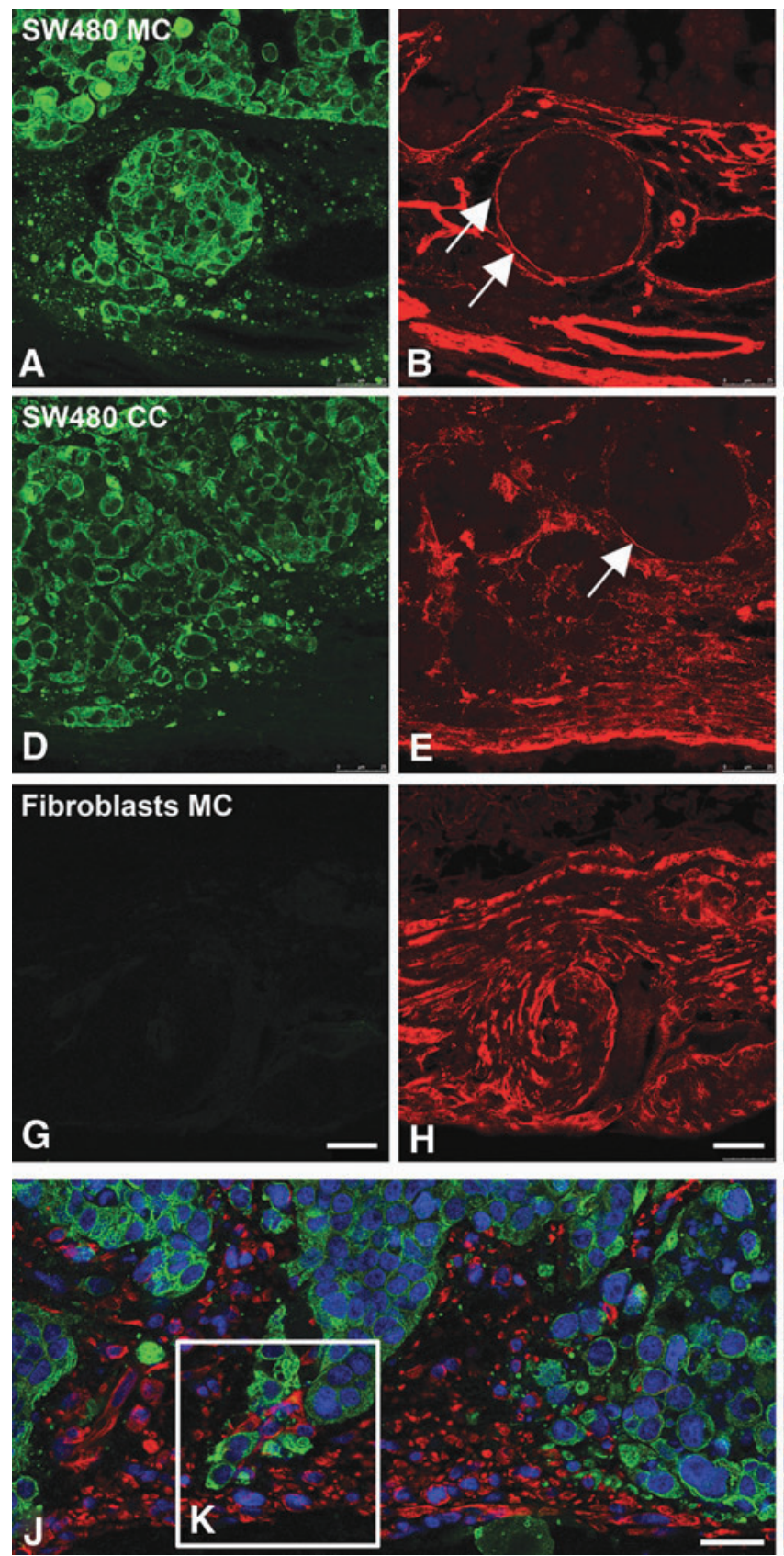
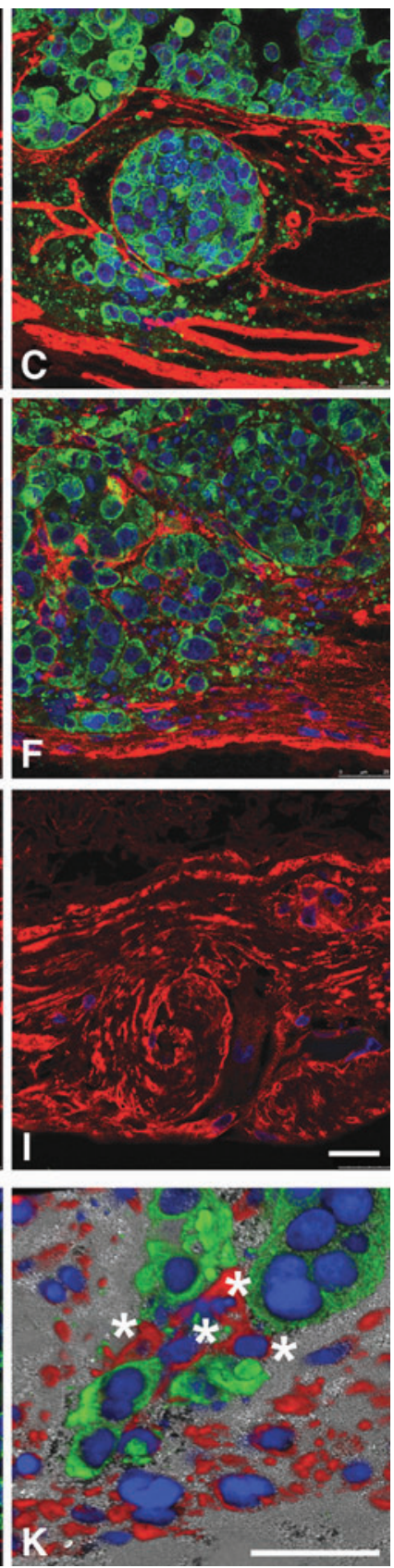

FIG. 9. In coculture with fibroblasts, the SISmuc scaffold is remodeled, and fibroblasts grow at the tip of tumor cells. The tumor cells alone (marked by PCK, green) do not remodel the collagen structure of the SISmuc (A-C). The basement membrane of the former crypts is clearly visible by immunohistochemical staining of collagen-IV (red in A-I) and is not interrupted (arrows in B). In coculture with fibroblasts (D-F), the distinct border of the former crypts is only partially visible (arrow in E) and the SISmuc is compacted with collagenIV (E, F). In monoculture, the fibroblasts remodel the SISmuc (G, H). In some areas of the coculture, fibroblasts (stained by vimentin, red in $\mathbf{J}, \mathbf{K}$ ) intermingle with the tumor cells (stained by PCK, green in $\mathbf{J}, \mathbf{K}$ ) and grow in front of them into the collagen structure of the SISmuc (K). Scale bars in (G-I) represent $25 \mu \mathrm{m}$ for (A-I). Scale bars in ( $\mathbf{J})$ and (K) represent $25 \mu \mathrm{m}$. Color images available online at www.liebertpub.com/tec treated with 5-FU (data not shown). In 2D, the coculturing of cells resulted in a fibroblast monolayer with single tumor cells placed on top and no tumor cell aggregates could be observed (Supplementary Fig. S2B, C).

\section{Discussion}

We developed a histologically complex 3D intestinal colon cancer model, including a tumor-stroma, on the basis of the biological collagen scaffold SISmuc, which has already been used for a lung tumor test system ${ }^{12}$ and which derives from the BioVaSc.

Our tissue-engineered 3D model reflects different aspects of tumors on morphological and molecular levels: (1) the formation of a differentiated monolayer of human $\mathrm{Caco} 2$ colon cancer cells in contrast to a more scattered growth pattern of SW480 cells accompanied by a loss of vimentin when cultured in $3 \mathrm{D}$, (2) a reduction of the proliferation correlative to clinical samples, (3) the induction of tumorlike structures of malignantly growing SW480 tumor cells by coculture with fibroblasts destroying basement membrane integrity that was not observed in $\mathrm{Caco} 2$ cocultures, and (4) the generation of a tight tissue-like association of tumor cells with each other and with the fibroblasts under dynamic culture conditions in a bioreactor. The resulting artificial tumor tissue with stroma resembles native adenocarcinomas and a clinically relevant treatment with 5-FU could be tested as a proof of principle.

By culturing tumor cells in 3D conditions on our matrix, their proliferation strongly decreases. Although an increase 

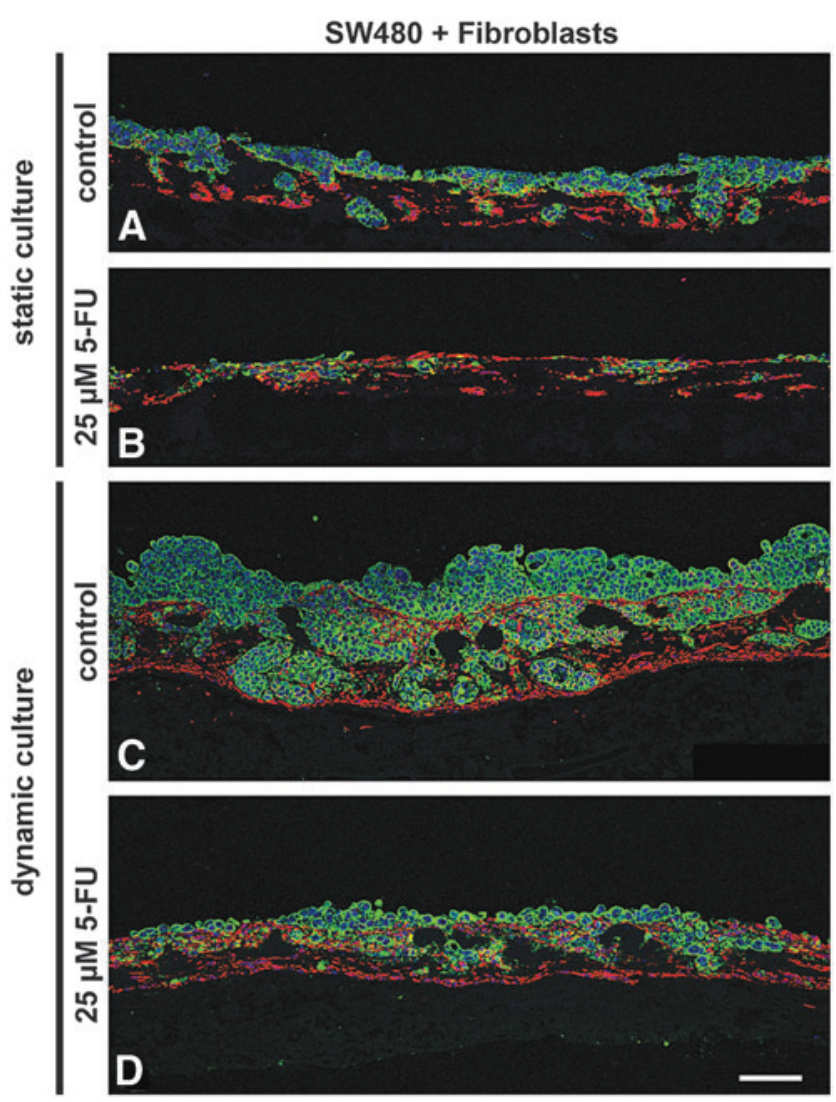

FIG. 10. Treatment of SW480 in coculture with fibroblasts with $25 \mu \mathrm{M}$ 5-FU under static as well as dynamic conditions. Under static culture conditions (A, B), treatment with 5-FU results in decreased tumor cell numbers (green) but leaves fibroblasts (red) unaffected (B). The same effect can be seen under dynamic conditions $(\mathbf{C}, \mathbf{D})$ where tumor cell numbers are much higher in the untreated control $(\mathbf{C})$ compared to the treated sample (D). Scale bar in D: $100 \mu \mathrm{m}$ for (A-D). PCK: green, vimentin: red, DAPI: blue. 5-FU, 5-fluorouracil. Color images available online at www.liebertpub.com/tec

in the percentage of proliferating cells can be seen in some metastases where the Ki67 index has been identified as a prognostic marker, ${ }^{18}$ the number of proliferating cells is usually lower in most tumors. Concerning cytostatic drugs, our model should thereby reduce false-positive results in preclinical testing that are thought to be derived at least, in part, from artificially high proliferation rates up to 90 or even $100 \%$ in $2 \mathrm{D}$ culture. ${ }^{19}$

In 1998, Kammerer et al. tested a similar decellularized human colonic matrix for the development of tumor models. ${ }^{20}$ They stressed the potential of decellularized tissue scaffolds as they included parts of the decellularized human colon as basis for tumor and stroma generation. However, they did not succeed with further developments. In the present study, we used a jejunal segment of porcine origin, which has several advantages. We developed a standardized tumor model based on the SISmuc, which is a nonvascularized part of the BioVaSc. This model can be vascularized in future settings by reseeding human endothelial cells into the preserved vessel structures as done before. ${ }^{21,22}$ The jejunal compartment is superior to colon tissue as it exhibits a higher vascularization and contains significantly less bacteria. This facilitates the decellularization process in bioreactors and the creation of a sterile scaffold, a fact of major importance as the use of sodium deoxycholate for decellularization hinders the addition of antibiotics. Moreover, the jejunum is much longer than the colon and thus fewer animals have to be sacrificed.

Since evolution has produced a high conservation of ECM proteins from porcine to human beings, it is even possible to implant such constructs into patients after recellularization of our scaffold with human cells. ${ }^{22}$ This indicates that our porcine matrix is suitable for human tumor model generation. Furthermore, porcine gut is easily obtainable from commercial pig breeders, which facilitates the production of pharmaceutical test systems.

Caco 2 cells are well established for applications in 3D cell culture models that simulate the intestinal barrier and are used for drug uptake studies. ${ }^{23,24}$ As shown before, they exhibit a differentiated phenotype in a suitable microenvironment. $^{25}$ To investigate to which extent our decellularized tissue scaffold reflects tumor characteristics-especially concerning invasion and metastasis-we focused on the more malignant SW480 cell line. In fact, the 3D microenvironment showed a stronger impact on SW480 cells than on $\mathrm{Caco} 2$ cells as marked by a downregulation of vimentin indicating MET induction-a hallmark for metastasis generation. ${ }^{9}$

Remarkably, coculture with fibroblasts induced only SW480 cells to form tumor-like cell clusters with fibroblasts within the matrix, whereas Caco2 cells grew on top of the matrix separated from the fibroblasts beneath. In some areas, we observed the generation of multilayers as found in hyperplastic epithelial regions: one of the first steps in tumorigenesis. This indicates different cell-specific interactions of both tumor cell types with the same fibroblast population and stresses the impact of signaling circuits between the tumorstroma and the tumor cells on tumor formation. It is widely recognized that the tumor-stroma plays a key role in tumor growth and invasion by the secretion of degrading enzymes and by storing cytokines. ${ }^{6,26}$ This changes signaling and thereby drug response in different invasive states. ${ }^{27}$ In fact, colon cancer tissue usually displays a very large proportion of fibrotic tissue. ${ }^{28,29}$ These issues further emphasize the importance to include the stroma component in preclinical tumor models.

To test the robustness of the system, different seeding conditions were compared such as different ratios of tumor cells to fibroblasts, different orders of seeding, first the fibroblasts and then the tumor cells or vice versa, as well as different donors of primary fibroblasts. Similar morphological results were observed in all the different experimental settings (data not shown). For standardization, we seeded both kinds of cells at the same time to generate the test model.

On a molecular level, we investigated the differentiation status and the degree of EMT by E-cadherin/ $\beta$-catenin and $\mathrm{PCK} /$ vimentin fluorescence double staining, respectively. In healthy epithelia, $\beta$-catenin is linked to E-cadherin at the cell border connecting it to the actin cytoskeleton. In primary tumors, E-cadherin expression is reduced and translocates to the cytoplasm at the invasion front. $\beta$-Catenin dissociates from E-cadherin and also translocates to the cytoplasm as well as into the nucleus. In this study, it 
induces the expression of other genes important for EMT and inhibitors of apoptosis, as reviewed concisely by Lee et $a .^{30}$ Nuclear localization of $\beta$-catenin correlates with a poor prognosis in the clinic. ${ }^{31}$ In colorectal carcinogenesis, many pro-oncogenic factors encourage $\beta$-catenin translocation from the adherens junction to the nucleus, and antioncogenic factors inhibit nuclear $\beta$-catenin signaling. This stresses its vital part in tumor development. ${ }^{32}$ When metastases extravasate into distant healthy tissues to form a macroscopic secondary tumor, cancer cells have to cross the basement membrane and revert to an epithelial state in a process that is called MET and a strong E-cadherin expression is found in these areas. ${ }^{33}$

Our 3D SW480 colon cancer model showed strong Ecadherin expression particularly in areas of dense tumor cell aggregate formation. This effect was more pronounced in a flow bioreactor under dynamic conditions enhancing close cell associations. Furthermore, we observed a strong downregulation of vimentin expression in 3D compared to 2D culture - under static as well as dynamic conditions. Since SW480 cancer cells are known to develop more epithelial characteristics at a higher confluency, ${ }^{34}$ this effect could be related to a higher confluency in the $3 \mathrm{D}$ cell culture. Thus, the induction of tighter cell to cell contacts in $3 \mathrm{D}$ cell culture conditions could trigger MET induction, as it takes place in tissues into which metastases originating from circulating single tumor cells grow. The MET process characterized by E-cadherin re-expression is thought to be a critical step during metastasis formation, as reviewed by Wells et al. ${ }^{33}$

As mentioned before, at least two different mechanisms exist for tumor cell invasion where fibroblasts play a central part. First, fibroblasts are important for single-cell invasion because this needs the EMT of tumor cells induced by myofibroblasts of the adjacent tumor-stroma. ${ }^{35,36}$ Second, they are also essential for collective invasion processes ${ }^{37}$ as they are able to remodel the scaffold and to guide tumor cells through this matrix. Accordingly, in deep layers of the collagen scaffold, we could observe patterns of fibroblasts in front of bulky tumor cell formations. In contrast to single-cell invasion, collective invasion is considered to be independent of EMT in tumor cells and to be associated with fibroblasts. The mesenchymal marker vimentin is not reactivated in SW480 cells in the coculture setting, indicating no EMT involvement in this process, thus supporting the hypothesis that collective invasion is taking place in our model.

As a conclusion, we could generate a model for a more advanced tumor stage for which only a limited number of models are available - even though such patients need the most support.

To show that our model is a useful tool in preclinical testing, we performed drug response analyses with 5-FU as a commonly applied drug in colon cancer treatment. ${ }^{38}$ In contrast to monoculture settings under $2 \mathrm{D}$ and $3 \mathrm{D}$ conditions, we could show that a 5-FU treatment decreased the number of tumor cells under static as well as dynamic 3D conditions in coculture with fibroblasts. A low sensitivity of SW480 cells to 5-FU has been reported in several studies before, ${ }^{39-41}$ even though this drug is a standard therapy in the clinic. This again stresses the tremendous importance of integrating stromal components into test systems to reveal reliable predictions correlating to the patients' situation in the clinic. In 2D cell culture, coculturing of SW480 cells and fibroblasts failed to generate close tumor-fibroblast aggregates and fibroblasts overgrew the tumor cells after a short culture period.

In the clinic, a mono-therapy-also in high plasma concentrations $^{42}$ - with 5-FU leads to a limited treatment response but in the end to unsatisfactory results in most cases. ${ }^{43}$ Therefore, 5-FU is applied mostly in combination with other substances such as folinic acid (FA) ${ }^{38,44-46}$ In future, other substances, for instance, a combination of 5-FU and FA, could be tested by using our artificial tissue, which is morphologically similar to colorectal adenocarcinoma metastases.

\section{Outlook}

This 3D model for colorectal tumors is directly available for drug testing since fundamental work has already established a standard operating protocol for lung tumor tissue generation and the quantitative measurement of drug responses regarding apoptosis and proliferation. ${ }^{12}$ In this model, apoptosis is quantified from supernatants by an M30 ELISA, which specifically recognizes epithelial cell apoptosis, thereby excluding fibroblasts. Double staining of Ki67 and the epithelial marker PCK enables the assessment of tumor cell proliferation in a coculture setting. This model can become more sophisticated by using the standardized biological scaffold BioVaSc-TERM ${ }^{\circledR},{ }^{14}$ which allows vascularization of tumor models. In serial bioreactors, this would also enable the investigation of the metastatic spread of single tumor cells into the artificial "blood" circulation following the process of MET, which is a critical step in metastasis.

SW480 colon cancer cells harbor an activating KRAS mutation that occurs in $30-40 \%$ of colorectal cancer, ${ }^{47}$ making them insensitive to epidermal growth factor receptor (EGFR) inhibitors. ${ }^{48}$ In the clinic, patients with these mutations are excluded from targeted therapies available against the EGFR. Therefore, novel therapeutic strategies are urgently needed for efficient intervention and could be gained by analysis of signaling changes on a targeted treatment in certain mutational backgrounds. These data could be integrated in bioinformatics network analyses as done before in the combined in vitro/in silico lung tumor model. ${ }^{12}$

\section{Conclusion}

In this study, we established a modular tissue-engineered 3D colon cancer model, including the adjacent tumor environment on a decellularized matrix. We were able to foster cell growth and metastasis-like tissue formation by coculturing fibroblasts and tumor cells in a newly developed bioreactor. This test system enables analyses of drug responses in advanced tumor stages, particularly of substances that target tumor-stroma interactions.

\section{Acknowledgments}

We thank Prof. Dr. Georg Krohne (Division of Electron Microscopy; Biocenter of the University of Wuerzburg) for the careful analysis of our SEM and TEM samples and discussion of the results, and Claudia Gehrig and Daniela Bunsen for their technical assistance. We also thank Matthias Schweinlin for explanting the porcine small intestine, 
Anna Schliermann for doing the HE stainings, and Saswati Sen for doing the villin stainings.

\section{Disclosure Statement}

No competing financial interests exist.

\section{References}

1. Bhattacharjee, Y. Biomedicine. Pharma firms push for sharing of cancer trial data. Science 338, 29, 2012.

2. Bissell, M.J., and Radisky, D. Putting tumours in context. Nat Rev Cancer 1, 46, 2001.

3. Hanahan, D., and Weinberg, R.A. Hallmarks of cancer: the next generation. Cell 144, 646, 2011.

4. Debnath, J., and Brugge, J.S. Modelling glandular epithelial cancers in three-dimensional cultures. Nat Rev Cancer 5, 675, 2005.

5. Fischbach, C., Chen, R., Matsumoto, T., Schmelzle, T., Brugge, J.S., Polverini, P.J., and Mooney, D.J. Engineering tumors with 3D scaffolds. Nat Methods 4, 855, 2007.

6. Kalluri, R., and Zeisberg, M. Fibroblasts in cancer. Nat Rev Cancer 6, 392, 2006.

7. Banyard, J., and Bielenberg, D.R. The role of EMT and MET in cancer dissemination. Connect Tissue Res 56, 403, 2015.

8. Friedl, P., Locker, J., Sahai, E., and Segall, J.E. Classifying collective cancer cell invasion. Nat Cell Biol 14, 777, 2012.

9. Gaggioli, C., Hooper, S., Hidalgo-Carcedo, C., Grosse, R., Marshall, J.F., Harrington, K., and Sahai, E. Fibroblast-led collective invasion of carcinoma cells with differing roles for RhoGTPases in leading and following cells. Nat Cell Biol 9, 1392, 2007.

10. Yao, D., Dai, C., and Peng, S. Mechanism of the mesenchymal-epithelial transition and its relationship with metastatic tumor formation. Mol Cancer Res 9, 1608, 2011.

11. Hoppensack, A., Kazanecki, C.C., Colter, D., Gosiewska, A., Schanz, J., Walles, H., and Schenke-Layland, K. A human in vitro model that mimics the renal proximal tubule. Tissue Eng Part C Methods 20, 599, 2014.

12. Stratmann, A.T., Fecher, D., Wangorsch, G., Gottlich, C., Walles, T., Walles, H., Dandekar, T., Dandekar, G., and Nietzer, S.L. Establishment of a human 3D lung cancer model based on a biological tissue matrix combined with a Boolean in silico model. Mol Oncol 8, 351, 2014.

13. Linke, K., Schanz, J., Hansmann, J., Walles, T., Brunner, H., and Mertsching, H. Engineered liver-like tissue on a capillarized matrix for applied research. Tissue Eng 13, 2699, 2007.

14. Schanz, J., Pusch, J., Hansmann, J., and Walles, H. Vascularised human tissue models: a new approach for the refinement of biomedical research. J Biotechnol 148, 56, 2010.

15. Jannasch, M., Groeber, F., Brattig, N.W., Unger, C., Walles, H., and Hansmann, J. Development and application of three-dimensional skin equivalents for the investigation of percutaneous worm invasion. Exp Parasitol 150, 22, 2015.

16. Moll, C., Reboredo, J., Schwarz, T., Appelt, A., Schurlein, S., Walles, H., and Nietzer, S. Tissue engineering of a human 3D in vitro tumor test system. J Vis Exp 78, 50460, 2013.

17. Biazik, J.M., Jahn, K.A., Su, Y., Wu, Y.N., and Braet, F. Unlocking the ultrastructure of colorectal cancer cells in vitro using selective staining. World J Gastroenterol 16, 2743, 2010.

18. Petrowsky, H., Sturm, I., Graubitz, O., Kooby, D.A., StaibSebler, E., Gog, C., Kohne, C.H., Hillebrand, T., Daniel,
P.T., Fong, Y., and Lorenz, M. Relevance of Ki-67 antigen expression and K-ras mutation in colorectal liver metastases. Eur J Surg Oncol 27, 80, 2001.

19. Cree, I.A., Glaysher, S., and Harvey, A.L. Efficacy of anticancer agents in cell lines versus human primary tumour tissue. Curr Opin Pharmacol 10, 375, 2010.

20. Kammerer, R., Ehret, R., and von Kleist, S. Isolated extracellular matrix-based three-dimensional in vitro models to study orthotopically cancer cell infiltration and invasion. Eur J Cancer 34, 1950, 1998.

21. Mertsching, H., Schanz, J., Steger, V., Schandar, M., Schenk, M., Hansmann, J., Dally, I., Friedel, G., and Walles, T. Generation and transplantation of an autologous vascularized bioartificial human tissue. Transplantation $\mathbf{8 8}$, 203, 2009.

22. Steinke, M., Dally, I., Friedel, G., Walles, H., and Walles, T. Host-integration of a tissue-engineered airway patch: two-year follow-up in a single patient. Tissue Eng Part A 21, 573, 2015.

23. Heinlein, A., Metzger, M., Walles, H., and Buettner, A. Transport of hop aroma compounds across Caco-2 monolayers. Food Funct 5, 2719, 2014.

24. Giusti, S., Sbrana, T., La Marca, M., Di Patria, V., Martinucci, V., Tirella, A., Domenici, C., and Ahluwalia, A. A novel dual-flow bioreactor simulates increased fluorescein permeability in epithelial tissue barriers. Biotechnol J 9, 1175, 2014

25. Pusch, J., Votteler, M., Gohler, S., Engl, J., Hampel, M., Walles, H., and Schenke-Layland, K. The physiological performance of a three-dimensional model that mimics the microenvironment of the small intestine. Biomaterials 32, 7469, 2011.

26. Ostman, A., and Augsten, M. Cancer-associated fibroblasts and tumor growth-bystanders turning into key players. Curr Opin Genet Dev 19, 67, 2009.

27. Liotta, L.A., and Kohn, E.C. The microenvironment of the tumour-host interface. Nature 411, 375, 2001.

28. Fleming, M., Ravula, S., Tatishchev, S.F., and Wang, H.L. Colorectal carcinoma: pathologic aspects. J Gastrointest Oncol 3, 153, 2012.

29. Peddareddigari, V.G., Wang, D., and Dubois, R.N. The tumor microenvironment in colorectal carcinogenesis. Cancer Microenviron 3, 149, 2010.

30. Lee, J.M., Dedhar, S., Kalluri, R., and Thompson, E.W. The epithelial-mesenchymal transition: new insights in signaling, development, and disease. J Cell Biol 172, 973, 2006.

31. Chen, Z., He, X., Jia, M., Liu, Y., Qu, D., Wu, D., Wu, P., Ni, C., Zhang, Z., Ye, J., Xu, J., and Huang, J. $\beta$-catenin overexpression in the nucleus predicts progress disease and unfavourable survival in colorectal cancer: a meta-analysis. PLoS One 8, e63854, 2013.

32. Wong, N.A., and Pignatelli, M. Beta-catenin-a linchpin in colorectal carcinogenesis? Am J Pathol 160, 389, 2002.

33. Wells, A., Yates, C., and Shepard, C.R. E-cadherin as an indicator of mesenchymal to epithelial reverting transitions during the metastatic seeding of disseminated carcinomas. Clin Exp Metastasis 25, 621, 2008.

34. Brabletz, T., Jung, A., Reu, S., Porzner, M., Hlubek, F., Kunz-Schughart, L.A., Knuechel, R., and Kirchner, T. Variable beta-catenin expression in colorectal cancers indicates tumor progression driven by the tumor environment. Proc Natl Acad Sci U S A 98, 10356, 2001.

35. Orimo, A., Gupta, P.B., Sgroi, D.C., Arenzana-Seisdedos, F., Delaunay, T., Naeem, R., Carey, V.J., Richardson, A.L., 
and Weinberg, R.A. Stromal fibroblasts present in invasive human breast carcinomas promote tumor growth and angiogenesis through elevated SDF-1/CXCL12 secretion. Cell 121, 335, 2005.

36. Radisky, D.C., Levy, D.D., Littlepage, L.E., Liu, H., Nelson, C.M., Fata, J.E., Leake, D., Godden, E.L., Albertson, D.G., Nieto, M.A., Werb, Z., and Bissell, M.J. Rac1b and reactive oxygen species mediate MMP-3-induced EMT and genomic instability. Nature 436, 123, 2005.

37. Friedl, P., and Wolf, K. Tube travel: the role of proteases in individual and collective cancer cell invasion. Cancer Res 68, 7247, 2008.

38. Herdrich, K., and Weinberger, H. Selected Schedules of Therapy for Malignant Tumors. Frankfurt am Main, Germany: Baxter Oncology, 2003.

39. Bracht, K., Nicholls, A.M., Liu, Y., and Bodmer, W.F. 5Fluorouracil response in a large panel of colorectal cancer cell lines is associated with mismatch repair deficiency. $\mathrm{Br}$ J Cancer 103, 340, 2010.

40. Yoshikawa, R., Kusunoki, M., Yanagi, H., Noda, M., Furuyama, J.I., Yamamura, T., and Hashimoto-Tamaoki, T. Dual antitumor effects of 5-fluorouracil on the cell cycle in colorectal carcinoma cells: a novel target mechanism concept for pharmacokinetic modulating chemotherapy. Cancer Res 61, 1029, 2001.

41. Mhaidat, N.M., Bouklihacene, M., and Thorne, R.F. 5Fluorouracil-induced apoptosis in colorectal cancer cells is caspase-9-dependent and mediated by activation of protein kinase C-delta. Oncol Lett 8, 699, 2014.

42. Bocci, G., Danesi, R., Di Paolo, A.D., Innocenti, F., Allegrini, G., Falcone, A., Melosi, A., Battistoni, M., Barsanti, G., Conte, P.F., and Del Tacca, M. Comparative pharmacokinetic analysis of 5-fluorouracil and its major metabolite 5-fluoro-5,6-dihydrouracil after conventional and reduced test dose in cancer patients. Clin Cancer Res 6, 3032, 2000.

43. de Vos tot Nederveen Cappel, W.H., Meulenbeld, H.J., Kleibeuker, J.H., Nagengast, F.M., Menko, F.H., Griffioen, G., Cats, A., Morreau, H., Gelderblom, H., and Vasen, H.F. Survival after adjuvant 5-FU treatment for stage III colon cancer in hereditary nonpolyposis colorectal cancer. Int $\mathbf{J}$ Cancer 109, 468, 2004.

44. Loehrer, P.J., Sr., Turner, S., Kubilis, P., Hui, S., Correa, J., Ansari, R., Stephens, D., Woodburn, R., and Meyer, S. A prospective randomized trial of fluorouracil versus fluorouracil plus cisplatin in the treatment of metastatic colorectal cancer: a Hoosier Oncology Group trial. J Clin Oncol 6, 642, 1988.

45. Douillard, J.Y., Cunningham, D., Roth, A.D., Navarro, M., James, R.D., Karasek, P., Jandik, P., Iveson, T., Carmichael, J., Alakl, M., Gruia, G., Awad, L., and Rougier, P. Irinotecan combined with fluorouracil compared with fluorouracil alone as first-line treatment for metastatic colorectal cancer: a multicentre randomised trial. Lancet 355, 1041, 2000.

46. Giacchetti, S., Perpoint, B., Zidani, R., Le Bail, N., Faggiuolo, R., Focan, C., Chollet, P., Llory, J.F., Letourneau, Y., Coudert, B., Bertheaut-Cvitkovic, F., LarregainFournier, D., Le Rol, A., Walter, S., Adam, R., Misset, J.L., and Levi, F. Phase III multicenter randomized trial of oxaliplatin added to chronomodulated fluorouracil-leucovorin as first-line treatment of metastatic colorectal cancer. J Clin Oncol 18, 136, 2000.

47. Vogelstein, B., Fearon, E.R., Hamilton, S.R., Kern, S.E., Preisinger, A.C., Leppert, M., Nakamura, Y., White, R., Smits, A.M., and Bos, J.L. Genetic alterations during colorectal-tumor development. N Engl J Med 319, 525, 1988.

48. Lievre, A., Bachet, J.B., Le Corre, D., Boige, V., Landi, B., Emile, J.F., Cote, J.F., Tomasic, G., Penna, C., Ducreux, M., Rougier, P., Penault-Llorca, F., and Laurent-Puig, P. KRAS mutation status is predictive of response to cetuximab therapy in colorectal cancer. Cancer Res 66, 3992, 2006.

Address correspondence to: Gudrun Dandekar, Dr.Sc.hum. Institute of Tissue Engineering and Regenerative Medicine (TERM)

University Hospital of the Julius-Maximilians University

Röntgenring 11

Würzburg 97070

Germany

E-mail: gudrun.dandekar@uni-wuerzburg.de

Received: December 17, 2015 Accepted: April 25, 2016

Online Publication Date: June 2, 2016 